\title{
Influence of the velocity gradient on the line formation in discs of cataclysmic variables
}

\author{
D. Korčáková ${ }^{1}$, T. Nagel ${ }^{2}$, K. Werner ${ }^{2}$, V. Suleimanov ${ }^{2,3}$, and V. Votruba ${ }^{1}$ \\ 1 Astronomical Institute of the Academy of Sciences of the Czech Republic, Fričova 298, 25165 Ondřejov, Czech Republic \\ e-mail: kor@sunstel.asu.cas.cz \\ 2 Institute for Astronomy and Astrophysics, Kepler Center for Astro and Particle Physics, Eberhard Karls University, Sand 1, \\ 72076 Tübingen, Germany \\ 3 Department of Astronomy, Kazan Federal University, Kremlevskaya 18, 420008 Kazan, Russia
}

Received 8 October 2010 / Accepted 23 January 2011

\section{ABSTRACT}

\begin{abstract}
Context. The velocity field gradient in the radiative transfer in disc models of cataclysmic variables (CVs) is usually neglected; however, the geometry of the system and the value of Keplerian velocity suggest that it can be important for high inclination angles. Aims. We investigate the influence of the Keplerian velocity gradient on the line formation in CV discs.

Methods. The vertical structure of the disc was determined using the NLTE accretion disc code $A c D c$, where the hydrostatic equation, the energy balance equation, the radiative transfer equation, the rate equations, and the equations of charge and particle conservation are consistently solved using the accelerated lambda iteration. NLTE opacities and emissivities are interpolated onto a 2D grid, where the radiative transfer equation was then solved with the velocity field taken into account.

Results. We show line-profile changes and limb-darkening dependences for the $\mathrm{H} \alpha$ and $\mathrm{H} \gamma$ lines in a model of SS Cyg, along with the HeI $4923 \AA$ line of a model representing a typical AM CVn system. Both systems are considered in the quiescent phase.

Conclusions. The line-profile changes due to the velocity gradient in the disc are small enough for most CV discs to allow the classical approach, where the radiative transfer is solved through the static disc and the velocity field is only taken into account in the final flux integration. However, the exact solution must be performed for CVs, where the disc rim plays an important role (inclination angles almost $90^{\circ}$ ). Extremely cool and relatively transparent discs under high inclinations should also be investigated with detailed radiative transfer models.
\end{abstract}

Key words. radiative transfer - methods: numerical - novae, cataclysmic variables - stars: individual: AM CVn stars: individual: SS Cyg

\section{Introduction}

Accretion discs of cataclysmic variables $(\mathrm{CVs})$ are a very complex phenomenon. It is necessary to consistently describe not only the structure of a disc, but also the transitional region, the hot spot, the central object, and wind originating in the disc. Moreover, the situation is sometimes complicated by the presence of a strong magnetic field. The construction of a consistent model of such complex objects is still beyond our capabilities. Individual works focus on particular problems, while other physical processes are only approximately taken into account. In the present paper we mainly discuss the subject of radiative transfer.

First models of accretion discs of $\mathrm{CV}$ s have been analytically based on the completely optically thin (Smak 1969) or thick (a black body - Bath 1974) approximation. Later, Schwarzenberg-Czerny \& Rózyczka (1977) calculated the radiation emanating from the disc using the LTE plane-parallel atmosphere models. The necessary values of $\log (g)$ and $T_{\text {eff }}$ were adopted from the disc structure. Kř́̌ž \& Hubený (1986) divided the disc into a set of concentric rings, where the vertical structure of the disc was determined by solving the hydrostatic, energy balance, and radiative transfer equations (see also Suleimanov 1992). They used the LTE approach, and it was later generalized to NLTE models (Wade \& Hubeny 1998; Nagel et al. 2004). For a multi-dimensional description, the Monte Carlo method is frequently used, which was first introduced for CVs by Knigge et al. (1995) for the investigation of spectral lines arising in accretion disc winds. The disadvantage of this method is the very slow convergence in the optically thick regions. A more general method that is able to describe optically thin as well as thick regions is the finite volume method (Adam 1990; Hummel et al. 1997). Including the wind in the models can be done in the Sobolev approximation (Sobolev 1946), improved for CVs by Rybicki \& Hummer (1983).

Here we investigate the influence of the velocity gradient on the radiative transfer through the disc, since this phenomenon is only considered in an approximate manner. The common approximation is to solve the radiative transfer through a static disc and to include the velocity field only in the final flux calculation. Another way is to use the Sobolev approximation (Sobolev 1946). Rybicki \& Hummer (1983) discussed the applicability of this approximation for $\mathrm{CV}$ discs in detail. Their conclusion disfavours it. Especially problematic are hydrogen lines, for which the thickness of velocity surfaces is comparable to the height of the discs, since the lines are very wide owing to Stark broadening.

The influence of the velocity gradient on the line-profile shapes of CVs, which are caused by the Keplerian motion in the disc, was first studied by Horne \& Marsh (1986). They constructed an analytical model that assumes Doppler broadening and neglects continuum opacity. They show the velocity shear 
becomes important when the inclination $i>60^{\circ}$. The optically thick lines change their profile shape from "U-like" to "V-like". Later, Marsh (1987) improved this model by including Stark broadening and continuum radiative transfer. Adam (1990) then solved the radiative transfer equation in $3 \mathrm{D}$ with the finite volume method. He included the velocity gradient using the observer's frame. The comoving frame for the description of the velocity field is used by Papkalla (1995), who solved this problem also in 3D using the ray-tracing method.

Due to the lack of the computer efficiency, these previous works had to use approximations for particular effects, which we are now able to treat more accurately. Even if Horne \& Marsh (1986) and Marsh (1987) did not use the Sobolev approximation, they did apply the escape probability method to solve the radiative transfer. Adam (1990), as well as later Papkalla (1995), used a two-level atom NLTE approximation with complete redistribution. They also assumed a constant value of density and temperature in the disc, which is far from reality.

Here, we present a model that is able to describe the emergent disc radiation with full velocity field taken into consideration, as well as the radial and tangential structure. The optical properties of the medium are interpolated from the accurate disc structure solutions along the vertical coordinate at different radii using the NLTE approach (Nagel et al. 2004). We use the accurate ray-tracing method for the radiation transfer equation in moving media (Korčáková \& Kubát 2005).

We focus particularly on models for SS Cyg and an AMCVn system. We choose SS Cyg, since even if there are a lot of observations and a lot of models applied, we do not understand the system well enough to describe the observed properties (Schreiber \& Lasota 2007). Smak (1981) found evidence of an additional emission source. Even if he used a simple model, problems with the explanation of the observed line profiles have also been encountered by Kromer et al. (2007). The used system of concentric rings is able to describe the observed spectra with high accuracy during the outburst, but the hydrogen lines are not reproduced very well during quiescence (Kromer et al. 2007).

In contrast to the SS Cyg disc, we also construct a model representing a disc typical of AMCVn stars. These systems are close, mass-transferring binaries with orbital periods shorter than $65 \mathrm{~min}$. This indicates that the donors must be compact objects, e.g., another white dwarf or a helium core burning star. Their spectra are dominated by helium lines, but do not show any sign of hydrogen. The discs presented here are geometrically very thin. Overview information about these interesting objects can be found, e.g., in Nelemans (2005) or Israel \& Dall'Osso (2009). We chose an AM CVn system in low-state for our study, since the discs in this case are more transparent and colder than in high state, which implies that the velocity field gradient could affect line profiles.

In the first part of this paper, we briefly describe the main properties and assumptions of our models. The validity of the applied technique is also discussed. The line-profile changes and limb-darkening dependences are shown in Chap. 3. A detailed discussion of our results is given in the last part of the paper (Chap. 4).

\section{Model description}

A consistent NLTE model of an accretion disc with a detailed description of the velocity field is still not possible with the current computation technique, so we chose the following approximative way. The hydrostatic structure, together with the opacity and source function, is calculated with the NLTE accretion disc code
AcDc (Nagel et al. 2004). The output from this code serves as input for the radiative transfer code (Korčáková \& Kubát 2005), which is used for the formal solution of the radiative transfer equation in the comoving frame.

\subsection{AcDc code}

For calculating metal-line blanketed NLTE accretion-disc models, we use the accretion-disc code $A c D c$ (Nagel et al. 2004). It is based on the radial structure of an $\alpha$-disc (Shakura \& Sunyaev 1973), assuming a stationary, geometrically thin disc (where total disc thickness is much smaller than the disc diameter). This allows decoupling of the vertical and radial structures and, together with the assumption of axial symmetry, we can separate the disc into concentric annuli of plane-parallel geometry. In that way, the radiative transfer becomes a $1 \mathrm{D}$ problem. Selfgravitation is neglected because the mass of the disc is far lower than the mass of the central object. The radial distribution of the effective temperature $T_{\text {eff }}$ can be described by

$T_{\mathrm{eff}}(R)=\left[\frac{3 G M_{1} \dot{M}}{8 \pi \sigma R^{3}}\left(1-\sqrt{\frac{R_{1}}{R}}\right)\right]^{1 / 4}$,

where $M_{1}$ and $R_{1}$ denote the mass and the radius of the central object, $\dot{M}$ is the mass-accretion rate, $G$ the gravitational constant, and $\sigma$ the Stefan-Boltzmann constant. The radial profile of surface mass density $\Sigma$ can be described by

$w \Sigma(R)=\frac{\dot{M}}{3 \pi}\left(1-\left(\frac{R_{1}}{R}\right)^{1 / 2}\right)$

with the kinematic viscosity $w$.

For each disc ring, the following set of coupled equations has to be solved simultaneously under the constraints of particle number and charge conservation:

- radiation transfer for the specific intensity $I$ at frequency $v$

$\mu \frac{\partial I(v, \mu, z)}{\partial z}=-\chi(v, z) I(v, \mu, z)+\eta(v, z)$,

with the absorption coefficient $\chi$, the emission coefficient $\eta$, the geometrical disc height $z$ above the midplane, and $\mu=$ $\cos \theta$ with $\theta$ is the angle between the ray and $z$;

- hydrostatic equilibrium between gravitation, gas pressure $P_{\text {gas }}$, and radiation pressure

$\frac{\mathrm{d} P_{\mathrm{gas}}}{\mathrm{d} m}=\frac{G M_{1}}{R^{3}} z-\frac{4 \pi}{c} \int_{0}^{\infty} \frac{\chi(v)}{\rho} H(v, z) \mathrm{d} v$,

where $H$ denotes the Eddington flux, and $\rho$ the mass density. Here we also introduced the column mass depth $m$ as

$m(z)=\int_{z}^{\infty} \rho\left(z^{\prime}\right) \mathrm{d} z^{\prime}$

- energy balance equation

$E_{\text {mech }}=E_{\text {rad }}+E_{\text {conv }}$.

The viscously generated energy $E_{\text {mech }}$ is equal to the radiative energy loss $E_{\text {rad }}$ with

$E_{\mathrm{rad}}=4 \pi \int_{0}^{\infty}(\eta(v, z)-\chi(v, z) J(v, z)) \mathrm{d} v=\frac{\mathrm{d} F}{\mathrm{~d} z}$ 


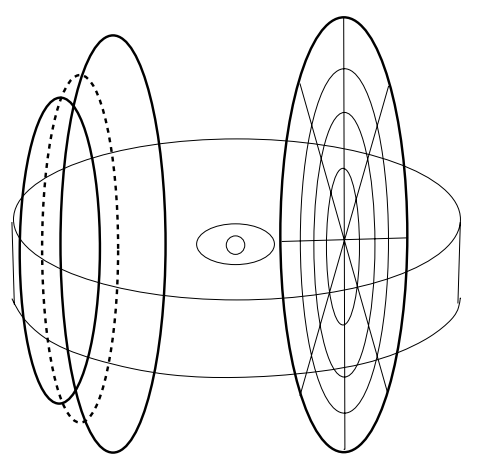

Fig. 1. Grid geometry. Solid lines indicate the "main" planes, where the radiative transfer is solved, while the additional planes for the flux calculation are depicted with dashed lines. A polar coordinate system is introduced in every plane.

and

$E_{\text {mech }}(m)=\frac{9}{4} \frac{G M_{\star}}{R^{3}} w(m) \rho$.

Here, $J$ denotes the mean intensity and $w(m)$ the depthdependent kinematic viscosity. For the prescription of the viscosity, we use the Reynolds number $R e$. This has the advantage that no further assumptions concerning first values of disc height and speed of sound have to be made, as would be necessary using the $\alpha$-prescription (e.g. Hubeny $\&$ Hubeny 1997). The convective energy term $E_{\text {conv }}$ is neglected in our models;

- NLTE rate equations for the population numbers $n_{i}$ of the atomic levels $i$

$\frac{\partial n_{i}}{\partial t}=0=n_{i} \sum_{i \neq j} P_{i j}-\sum_{j \neq i} n_{j} P_{j i}$,

where $P_{i j}$ denotes the rate coefficients, consisting of radiative and collisional components.

By integrating the spectra of the individual annuli, one obtains a complete disc spectrum for different inclination angles, where the spectral lines are Doppler shifted according to the radial component of the Kepler rotational velocity. The input parameters that we have to provide for calculating disc rings are the mass $M_{1}$ and radius $R_{1}$ of the central object, the mass-accretion rate $\dot{M}$, the radius of the annulus $R$, the Reynolds number $R e$, and the chemical abundances. Detailed information about the involved atomic data is provided in the form of a model atom (cf. Rauch \& Deetjen 2003).

\section{2. $2.5 D$ radiative transfer model}

We do not perform the last step, which is to integrate the specific intensity, using the $A c D c$ code here. Instead, the final formal solution of the radiative transfer equation is calculated using the 2.5D radiative transfer code Korčáková \& Kubát (2005).

The main assumption of this model is axial symmetry. This allows solving not a complete 3D problem, but instead the radiative transfer equation independently in planes intersecting the object (Fig. 1). To achieve a sufficient description of this grid for the flux calculation, it is necessary to have at least three hundred planes for objects, where the velocity reaches high values (about $2000 \mathrm{~km} \mathrm{~s}^{-1}$ ), e.g. hot stars with wind or CVs. However, it is not necessary to solve the transfer problem on such a fine grid, since line profiles in the rest frame connected with the medium do not change significantly. Therefore, the radiative transfer equation is solved only on a sparse grid (from forty to eighty planes depending on media properties), and the interpolation to the other planes is performed for the flux. The radiative transfer equation is solved using a combination of long (Cannon 1970) and short characteristics (Kunasz \& Auer 1988) methods, through the whole object structure; i.e., the central white dwarf (WD), the transition region, the disc, as well as the wind region can be considered.

The velocity is included in the solution using the comoving frame. Generally, it is possible to combine the radial velocity (representing stellar winds) with the tangential velocity distribution (stellar rotation). For our purpose now, we only assume a rotating disc where the media follows the Keplerian law

$v\left(r, \theta_{\pi / 2}\right)=\left(g M_{1} / r\right)^{1 / 2}$.

Latitude dependence is also permitted:

$v(r, \theta)=v\left(r, \theta_{\pi / 2}\right) \sin (\theta)$.

Since the velocity gradient between neighbouring cells is small, only the Doppler shift is considered. The transformation of the intensity is neglected. The intensity transformation is also neglected in the flux calculation. The intensity ratio in the most internal parts in the disc of the AM CVn system (maximal rotation velocity is $3360 \mathrm{~km} \mathrm{~s}^{-1}$ ) is $\sim 0.97$ or $\sim 1.03$, depending on the position in the disc. Concerning the Kepler velocity law and its Doppler projection to the observer, such extreme velocity values occur only in a small region. Omitting the intensity transformation can affect the line-profile shape a little bit, however, it has a negligible influence on the amplitude of the shear effect.

In comparison with the original model developed for hot stars, we introduce the possibility of integrating the final outgoing flux not only at the boundary of the grid, but also at the disc boundary, since it reduces the grid requirements. The integration of the flux at the disc boundary is only possible in the case of an optically thin corona. In this work, we neglect the coronal contribution to better evaluate the influence of the velocity gradient on the line formation. For the same reason, the radiation from the WD is also omitted.

\subsection{Model justification}

The applicability of the model described above is limited especially by two factors: the axial symmetry approximation and the nonrelativistic velocity field.

\section{axial symmetry approach}

The axial symmetry is broken, above all, by the presence of a hot spot. Nevertheless, spiral arms can also play an important role. However, these effects require a complete 3D description. In this case, including the velocity field in the radiative transfer equation will be very problematic for the computation requirements.

\section{velocity field limits}

There are two limits that must be respected. The first is the sufficient description of the frequency grid. Since the transformation of the frequency due to the Doppler shift is performed at the cell boundaries, the reasonable description of the line must be conserved. The Doppler shift due to the macroscopic motion must be less than a quarter of the Doppler half width. Failing this, 
Table 1. Parameters of the individual rings of the SS Cyg disc model.

\begin{tabular}{lcccccc}
\hline \hline Ring & $T_{\text {eff }}[\mathrm{K}]$ & $\dot{M}\left[M_{\odot} / \mathrm{yr}\right]$ & $r[\mathrm{~cm}]$ & $\tau_{\text {Ros }}$ & $R e$ & $h[\mathrm{~cm}]$ \\
\hline 1 & 13391 & $1 \times 10^{-11}$ & $1 \times 10^{9}$ & 104 & $3 \times 10^{4}$ & $2.74 \times 10^{7}$ \\
2 & 9931 & $1 \times 10^{-11}$ & $2 \times 10^{9}$ & 125 & $3 \times 10^{4}$ & $5.41 \times 10^{7}$ \\
3 & 6258 & $1 \times 10^{-11}$ & $4 \times 10^{9}$ & 1.765 & $3 \times 10^{4}$ & $1.14 \times 10^{8}$ \\
4 & 4615 & $1 \times 10^{-11}$ & $6 \times 10^{9}$ & 0.360 & $3 \times 10^{4}$ & $1.85 \times 10^{8}$ \\
5 & 4826 & $5 \times 10^{-11}$ & $1 \times 10^{10}$ & 0.483 & $3 \times 10^{4}$ & $3.43 \times 10^{8}$ \\
6 & 4463 & $3 \times 10^{-10}$ & $2 \times 10^{10}$ & 0.312 & $5 \times 10^{3}$ & $9.58 \times 10^{8}$ \\
7 & 4379 & $1 \times 10^{-9}$ & $3 \times 10^{10}$ & 0.377 & $1 \times 10^{4}$ & $1.46 \times 10^{9}$ \\
8 & 4106 & $2 \times 10^{-9}$ & $4 \times 10^{10}$ & 0.287 & $1 \times 10^{4}$ & $2.10 \times 10^{9}$ \\
\hline
\end{tabular}

Notes. Values of effective temperature $T_{\text {eff }}$, mass accretion rate $\dot{M}$, distance $r$ from the white dwarf centre, the total Rosseland optical depth $\tau_{\text {Ros }}$, and Reynolds number $R e$. The vertical thickness $h$ of the rings measured from the disc midplane is written in the last column.

the grid must be finer. This is an essential complication, since it increases the computation requirements.

In the high-velocity limit, another difficulty arises. When relativistic effects start to be strong, the ray along which the radiative transfer equation is solved becomes distorted. Mihalas et al. (1976) showed that the aberration and advection terms can be neglected for stellar winds, where the ratio of the matter velocity and speed of light is typically $\sim 0.01$ or lower. In our case, the maximal rotation velocity reaches $3360 \mathrm{~km} \mathrm{~s}^{-1}$, which allows the classical approach.

\subsection{Model of SS Cyg during quiescence phase}

To construct the radial disc structure we use the parameters of the system determined by Bitner et al. (2007). From a series of spectroscopic and photometric observations as well as previous works the WD mass $M_{1}=0.81 \pm 0.19 M_{\odot}$ (we adopt the value $0.85 M_{\odot}$ ), they derived the mass ratio of the secondary $\mathrm{K}$ component and WD $q=M_{2} / M_{1}=0.683 \pm 0.012$, and the separation of the mass centres $a=(1.36 \pm 0.11) \times 10^{11} \mathrm{~cm}$. The orbital period of the system is $P_{\text {orb }}=0.27512973$ days. From these parameters we can roughly determine the geometry of the system following Hellier (2001, Chap. 2.4) - the distance of the Lagrangian point from the primary white dwarf $R_{\mathrm{L}_{1}}=7.31 \times 10^{10} \mathrm{~cm}$, the minimal radius of the disc's outer edge $r_{\text {circ }}=1.37 \times 10^{10} \mathrm{~cm}$, and the maximal radius of the disc is determined by the tidal interaction of the secondary $r_{\text {tidal }}=4.85 \times 10^{10} \mathrm{~cm}$. The WD radius $\left(0.759 \times 10^{9} \mathrm{~cm}\right)$ is calculated using Wood (1995) tables assuming $T_{\text {eff }}=40000 \mathrm{~K}$.

All these parameters are necessary for constructing the geometrically thin $\alpha$-disc (Shakura \& Sunyaev 1973). This structure is taken as initial conditions for the $A c D c$ code. The disc is modeled by a set of eight concentric rings with different mass accretion rate (Table 1$)$. For our purpose, we include only hydrogen and helium in the calculations (41 NLTE and 21 LTE levels). The obtained opacity and emissivity for the $\mathrm{H} \alpha$ and $\mathrm{H} \gamma$ lines are plotted in Figs. 2 and 3, respectively.

\subsection{Model of an AMCVn disc during quiescence phase}

We also investigate the disc characterizing some AM CVn type system in the low state. These systems typically have a very low mass-accretion rate $\left(\sim 10^{-11} M_{\odot} / \mathrm{yr}\right)$ compared to AM CVn stars in the high state $\left(\sim 10^{-8} M_{\odot} / \mathrm{yr}\right)$. Their orbital period is in the range $40 \mathrm{~min}<P_{\text {orb }}<65 \mathrm{~min}$. Their spectra show emission
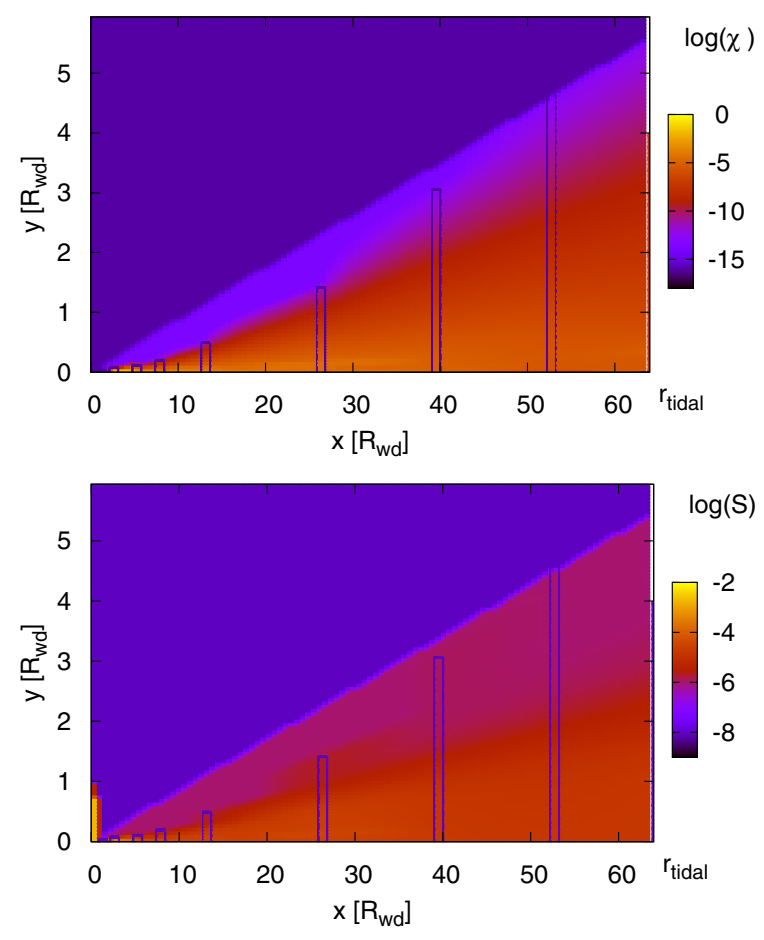

Fig. 2. Opacity and source function in the centre of the $\mathrm{H} \alpha$ line for the SS Cyg disc model. The system of concentric rings, which is used for the AcDc (Nagel et al. 2004) code, are depicted by rectangles. The opacity and emissivity in the rectangles correspond to the value from the $A c D c$ code. The tidal radius $r_{\text {tidal }}$ is depicted in the figure.

lines, especially strong are helium lines. We choose a low-state AM CVn system, since the discs are relatively cold and optically thin in these objects, and some helium lines are much narrower than hydrogen lines in classical, hydrogen-rich CVs. Both facts indicate that the velocity shear effect can be important in this case.

The model of the disc is adopted from Nagel et al. (2009). We assume $M_{1}=0.8 M_{\odot}, M_{2}=0.05 M_{\odot}, \dot{M}=10^{-11} M_{\odot} / \mathrm{yr}$, and $P_{\text {orb }}=3000 \mathrm{~s}$. From these basic parameters following Hellier (2001, Chap. 2.4) and the third Kepler law, we obtain additional system parameters: $a=2.95 \times 10^{10} \mathrm{~cm}, R_{\mathrm{L} 1}=2.28 \times 10^{10} \mathrm{~cm}$, $R_{\text {circ }}=6.38 \times 10^{9} \mathrm{~cm}$, and $R_{\text {tidal }}=1.66 \times 10^{10} \mathrm{~cm}$, while $R_{1}=$ $7.38 \times 10^{8} \mathrm{~cm}$ is taken from the Wood (1995) tables and $R e$ is set to $1 \times 10^{5}$.

The $\alpha$-disc is constructed based on these parameters and the vertical structure is computed with $A c D c$. Hydrogen, helium, carbon, nitrogen, oxygen, and silicon are included in calculations. 439 NLTE and 448 LTE levels are used for the atoms and ions description. The disc is divided into a set of nine rings (Table 2), where opacity and source function are obtained. The values interpolated in the 2D grid are plotted in Fig. 4.

\section{Results}

The opacity and source function distribution in the disc (Figs. 24), and the Keplerian velocity field (Eq. (10) and its latitudinal dependence (Eq. (11)) are used to solve the radiative transfer (Sect. 2.2). The numerical properties of these calculations are displayed in Table 3 . The frequency grid $\left(N_{v}\right)$ is determined by the opacity profile and velocity gradient, therefore it is different for each model. The number of radial $\left(N_{r}\right)$ and tangential points $\left(N_{\theta}\right)$ per quadrant are tabulated there. The number of tangential points per quadrant above the disc is written in the round 
D. Korčáková et al.: Influence of the velocity gradient on the line formation in discs of CVs
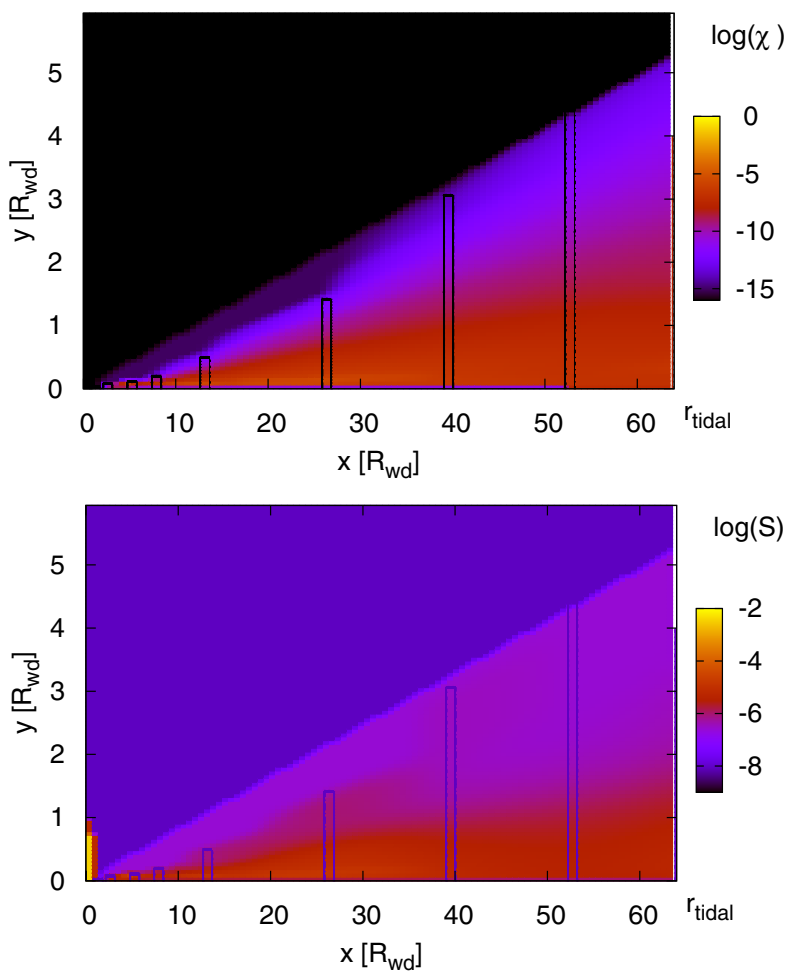

Fig. 3. Opacity and source function dependence in the disc model of SS Cyg. The figure is plotted for the centre of the $\mathrm{H} \gamma$ line. Rectangles show the original value from the $A c D c$ code.

Table 2. Parameters of the individual rings of our AM CVn disc model.

\begin{tabular}{lcccc}
\hline \hline Ring & $T_{\text {eff }}[\mathrm{K}]$ & $r[\mathrm{~cm}]$ & $\tau_{\text {Ros }}$ & $h[\mathrm{~cm}]$ \\
\hline 1 & 4890 & $7.4 \times 10^{8}$ & $4.5 \times 10^{-2}$ & $6.70 \times 10^{6}$ \\
2 & 12100 & $1 \times 10^{9}$ & 2.7 & $9.04 \times 10^{6}$ \\
3 & 10060 & $1.7 \times 10^{9}$ & $8.5 \times 10^{-1}$ & $1.61 \times 10^{7}$ \\
4 & 8100 & $2.5 \times 10^{9}$ & $1.9 \times 10^{-1}$ & $2.32 \times 10^{7}$ \\
5 & 6260 & $3.9 \times 10^{9}$ & $4.1 \times 10^{-2}$ & $3.47 \times 10^{7}$ \\
6 & 5100 & $5.2 \times 10^{9}$ & $2.9 \times 10^{-2}$ & $4.65 \times 10^{7}$ \\
7 & 4200 & $7.1 \times 10^{9}$ & $1.6 \times 10^{-2}$ & $6.70 \times 10^{7}$ \\
8 & 3600 & $9 \times 10^{9}$ & $1.0 \times 10^{-2}$ & $8.89 \times 10^{7}$ \\
9 & 3400 & $1 \times 10^{10}$ & $8.5 \times 10^{-3}$ & $1.01 \times 10^{8}$ \\
\hline
\end{tabular}

Notes. Values of effective temperature $T_{\text {eff }}$, distance $r$ from the centre of the white dwarf, the total Rosseland optical depth $\tau_{\text {Ros }}$, and disc thickness $h$.

brackets. It is not necessary to have a fine description in this region, since we consider no medium there. The angular description of the specific intensity is the same for all models. The real description depends on the number of planes and position in the disc, and it is more than one order higher. The last row shows the number of intersecting planes ( $\left.N_{\text {planes }}\right)$, where the radiative transfer is solved. The used system of planes for the flux calculation is written in round brackets. The set of concentric rings, where the vertical structure is obtained using the $A c D c$ code, consists of eight rings for SS Cyg and nine rings for AM CVn. These ring numbers are at the lower limit considering what is commonly used. Depending on the physical processes included in the modelling, a set of eight up to 35 rings is usually introduced. For instance, Nasser (2001) uses a set of eight rings for the spectra calculations of AM CVn systems. Since we only investigate the influence of the rotation velocity gradient and not other effects, our description of the disc structure appears to be sufficient.
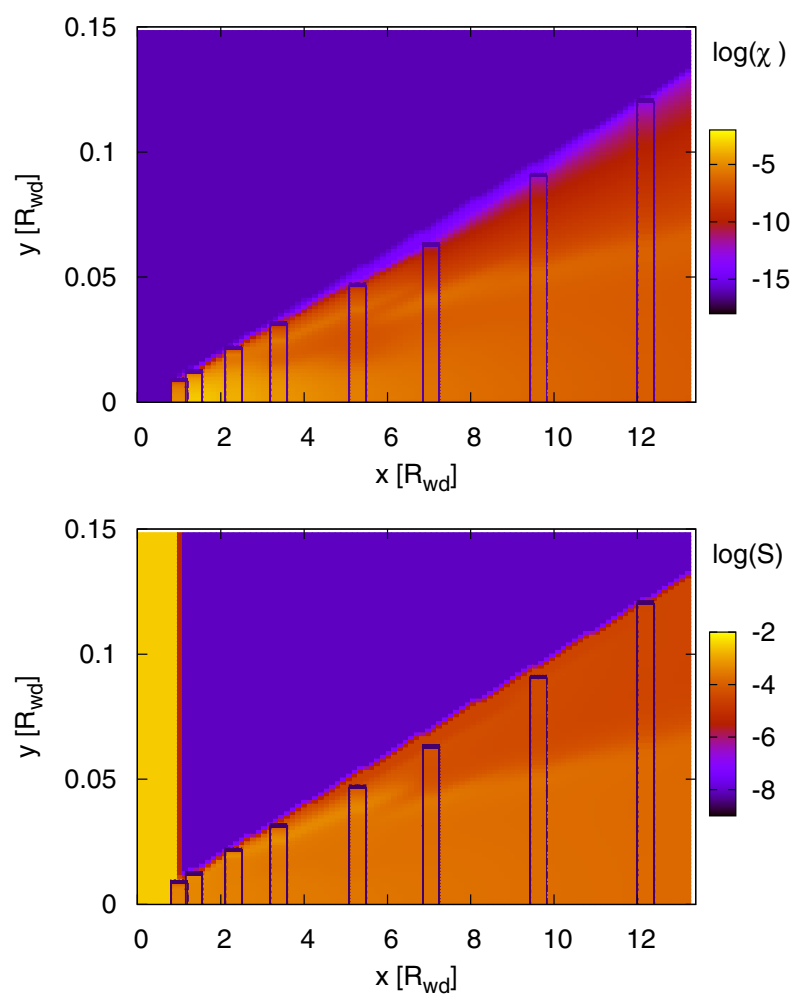

Fig. 4. Opacity and source function in the disc model of an AMCVn system. The values are plotted for the centre of the HeI $4923 \AA$ line. Rectangles denote values obtained using the $A c D c$ code. To illustrate the disc geometry, the white dwarf is depicted here (yellow "rectangle" in the right graph).

Table 3. Numerical properties of the $2.5 \mathrm{D}$ radiation transfer models.

\begin{tabular}{lccc}
\hline \hline & SS Cyg, $\mathrm{H} \alpha$ & SS Cyg, $\mathrm{H} \gamma$ & AMCVn, HeI \\
\hline$N_{v}$ & 381 & 267 & 457 \\
$N_{r}$ & 119 & 93 & 194 \\
$N_{\theta}$ & $81(15)$ & $88(15)$ & $80(15)$ \\
$N_{\mu}$ & 9 & 9 & 9 \\
$N_{\text {planes }}$ & $75(223)$ & $75(223)$ & $75(371)$ \\
\hline
\end{tabular}

Notes. Number of frequency points $N_{v}$, radial points $N_{r}$, tangential points $N_{\theta}$ (tangential points above the disc), angular description for specific intensity per quadrant $N_{\mu}$, planes $N_{\text {planes }}$ where the radiative transfer is solved, and planes used for the flux calculation (in round brackets).

The resulting line profiles and limb darkening effect are described in more detail in the following sections.

\subsection{Line profile shapes}

Line profiles from the discs are plotted in Figs. 5-7. To see the influence of the velocity gradient on the line formation, we also calculated the case where the radiative transfer is solved through the static disc and the velocity is only included in the final flux calculation. The results in Figs. 5-7 are plotted for different angles of view $-0^{\circ}$ is the face-on view and $90^{\circ}$ edge-on view. The upper right panel in each figure is the view just above the disc edge.

The influence of the velocity field on the line formation depends on the ratio of the value of frequency shift due to the macroscopic motion and the line width and also on the transparency of the medium in the region of a high-velocity gradient. Table 4 summarizes the maximal optical depth through the 


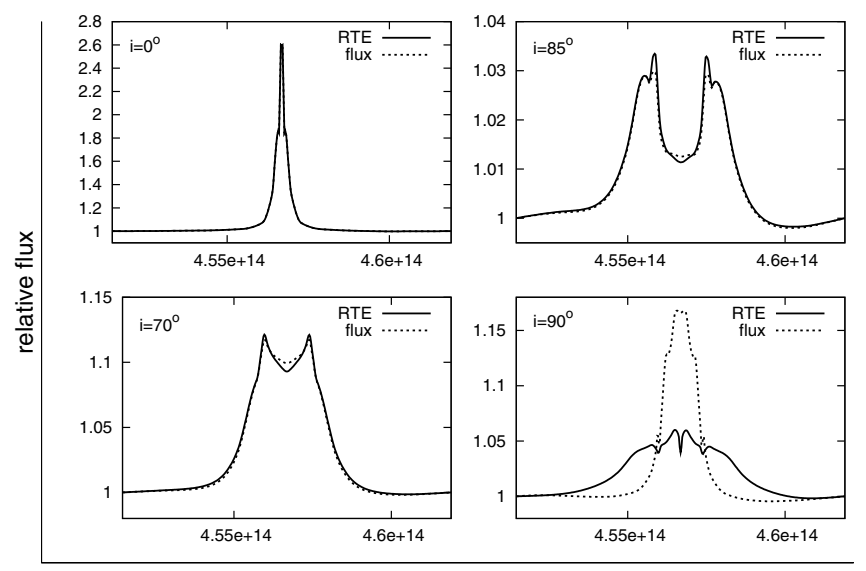

$v[\mathrm{~Hz}]$

Fig. 5. Relative flux of the $\mathrm{H} \alpha$ line in the SS Cyg model. The solid line indicates the solution where the velocity gradient is included in the radiative transfer. The dashed line denotes the solution where the radiative transfer is solved through a static disc, and the velocity field is only taken into account in the final flux calculation. The results are plotted for four inclination angles.

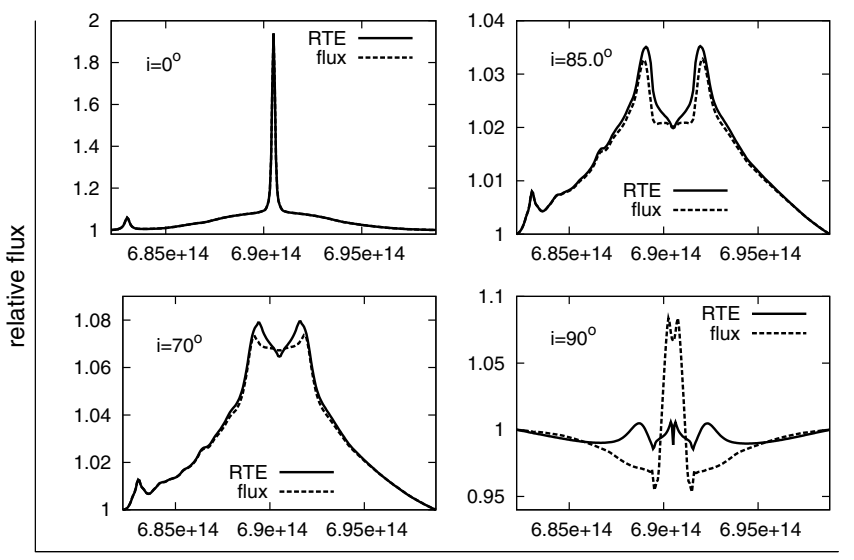

$v[\mathrm{~Hz}]$

Fig. 6. $\mathrm{H} \gamma$ line profiles from the SS Cyg model. The notation is the same as in Fig. 5.

Table 4. Optical depth in the line centre and continuum through the disc.

\begin{tabular}{lccc}
\hline \hline & SS Cyg, H $\alpha$ & SS Cyg, H $\gamma$ & AM CVn, HeI 4923 $\AA$ \\
\hline$\tau_{\text {line centre }}$ & $5 \times 10^{7}$ & $2 \times 10^{6}$ & $4 \times 10^{5}$ \\
$\tau_{\text {continuum }}$ & $6 \times 10^{4}$ & $3 \times 10^{5}$ & $1 \times 10^{2}$ \\
\hline
\end{tabular}

equatorial plane of the disc in the centre of the line and continuum in these three particular cases. In agreement with this, the difference between the accurate solution and the solution where the velocity is taken into account only in the final flux calculation is higher for the optically thinner $\mathrm{H} \gamma$ line than for the $\mathrm{H} \alpha$ line in the SS Cyg model. However, the difference at the face-on view (all panels in Figs. 5-7, except $i=90^{\circ}$ ) is negligible for observational accuracy. An important difference can be seen just above the disc edge in the case of an AMCVn system (Fig. 7, $i=89^{\circ}$ ). The disc is optically thinner there, but also the profile of the He line in the rest frame of medium is narrow. The velocity gradient is more comparable to the half-width of the line so it starts to affect the solution.
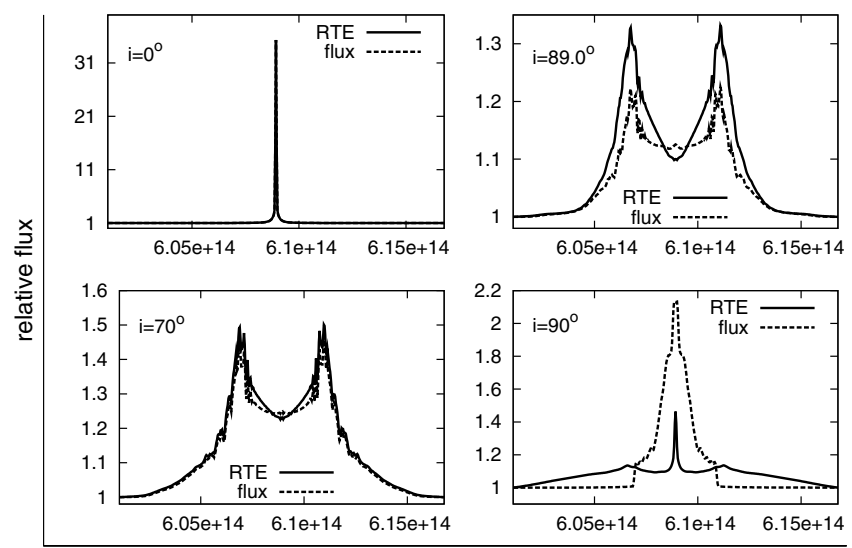

$v[\mathrm{~Hz}]$

Fig. 7. HeI $4923 \AA$ line profiles of an AM CVn model. The notation is the same as in Fig. 5.

The last panel of Figs. 5-7 shows the edge-on view $\left(i=90^{\circ}\right)$. There is a huge difference in the line-profile shapes for the AMCVn system, as well as for SS Cyg. This is caused by the specific intensity integration. The velocities from the inner part of the disc to the outer boundary are included in the integration for the edge-on view. Nevertheless, this is not the case for the rim. For the edge-on view, the integration along the rim boundary includes only the value of the rotation velocity at the rim, which is the lowest one. To reflect all the velocity distribution in this case, the velocity must be included in the radiative transfer. Even if the medium is optically thick at this angle, the line profile can be affected by the velocities in deep inner regions, since the line thermalization occurs in deeper regions than in the static case due to the Doppler shift.

The top right panels in Figs. 5-7 give the view just above the disc. The shear effect is most important in these high inclination angles. The difference for the $\mathrm{H} \alpha$ line of SS Cyg is negligible (Fig. 5), but the other two cases (Figs. 6 and 7) show an important difference. The emission heights are not only higher, in agreement with the higher escape probability due to the velocity gradient, but the line-profile shapes also change. Those results where the velocity is only taken into account in the flux calculation (dashed lines) show a classical "U" shape in the central parts. The velocity gradient of the medium changes this " $U$ " profile to the "V-like" shape. This agrees with the previous results of Horne \& Marsh (1986), Marsh (1987), and Papkalla (1995).

\subsection{Angular dependence of the flux}

Outgoing radiation from the disc strongly depends on the angle of view. This relation is caused by a geometrical effect, since the optical depth along the line-of-sight differs very much. The description of this limb-darkening effect is complicated, especially for accretion discs, since the medium is very anisotropic there. On the other hand, it provides a good possibility for testing our knowledge about the accretion disc structure. However, the statistical studies based on observations (Warner 1986, 1987; Morales-Rueda \& Marsh 2002; or Puebla et al. 2007) are not at sufficient accuracy. Above all, this comes from the uncertainty in determining the inclination angle and the fact that only objects with similar properties can be compared.

Nevertheless, we show the limb-darkening effect in Fig. 8 for our three cases. Since the radiative transfer is solved through the whole structure of the disc in our model, this effect is described 
SS Cyg, $\mathrm{H} \alpha$

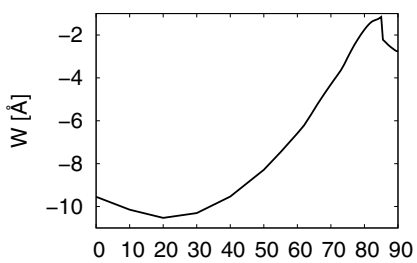

i $\left.{ }^{\circ}\right]$

$$
\text { SS Cyg, } \mathrm{H} \gamma
$$

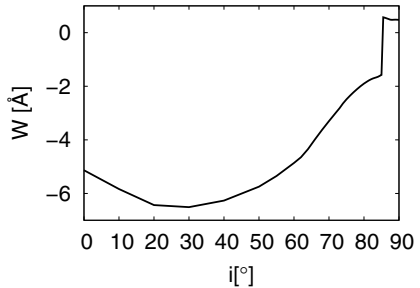

an AM CVn system, HeI $4923 \AA$

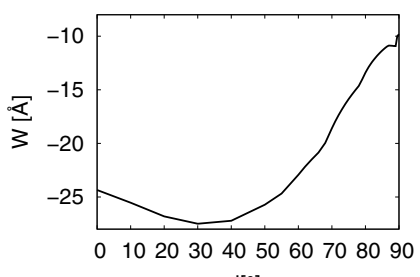

$\left.\mathrm{i}^{\circ}\right]$

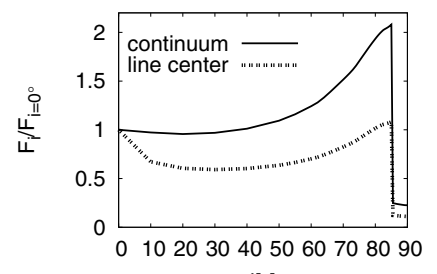

i[ $\left.{ }^{\circ}\right]$

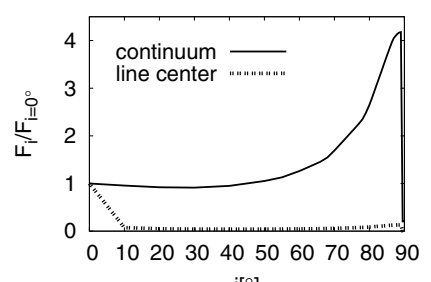

$\left.i^{\circ}\right]$
Fig. 8. Angular dependence of the equivalent width and flux in the continuum and line centre. Flux values are plotted relative to the flux at $i=0^{\circ}$ (face-on view).

very well in our case. We would specially like to point to the parts of the graphs in high inclination angles, where it is very problematic to achieve a good description using $1 \mathrm{D}$ models. The flux dependences on the inclination angle are plotted in the right hand panels of Fig. 8. Flux is displayed relative to the value for $i=0^{\circ}$. The dependence of the equivalent width is depicted in the left panels.

\section{Discussion and conclusions}

We study the influence of the velocity gradient, determined by the Keplerian law, on the line formation in the disc of CVs. The size of this effect depends on i) the value of frequency shift caused by the macroscopic motion relative to the line width; ii) the transparency of medium in regions of high velocity gradient; iii) the geometrical properties - the inclination angle and the disc opening angle.

The previous works of Horne \& Marsh (1986), Marsh (1987) and Adam (1990), Papkalla (1995) show, that the velocity shear changes the line-profile shapes from "U-like" to "V-like". We confirm this result (Figs. 5-7 left bottom and right top panels). However, the effect is stronger in their calculations than in our case. Previous results proved the importance of the velocity shear in the line profiles for inclination angles higher than $60^{\circ}$. In our case, there is an almost invisible difference between the solutions when i) the gradient of the velocity field is included in the solution of the radiative transfer equation and when ii) the radiative transfer is solved through the static disc and the velocity is only taken into account in the flux calculation.
This disagreement can come from following reasons. Firstly, Horne \& Marsh (1986), Adam (1990), and Papkalla (1995) used only the thermal Doppler broadening to compute line profiles. This supports the influence of the velocity gradient, since the ratio between the frequency shift due to the macroscopic motion and line width becomes larger. Marsh (1987) included Stark broadening for the study of $\mathrm{Z}$ Cha, but he adopted a rather low mass accretion rate, so that the effective temperature varies from $7000 \mathrm{~K}$ to $1000 \mathrm{~K}$. The line profiles in the comoving frame are narrow in this case, which increases the velocity shear effect. Nevertheless, these temperature values are too low for many of the CV discs. Adam (1990), and Papkalla (1995) have solved the radiative transfer through the disc in $3 \mathrm{D}$. However, they adopted a homogeneous disc, which is probably far from the reality. The density values vary through the disc by several orders of magnitude. The temperature also varies significantly in both radial and latitudinal directions. If the outgoing radiation only comes from a small part of the disc, not every velocity value affects the line profile significantly. For instance, the adopted structure of the disc in our cases gives such value of the source function and opacity, that most of the radiation in the line centre comes from the internal parts of the disc.

Our model describes the structure of the disc and the NLTE opacity and emissivity of the medium much more precisely. Solution of the radiative transfer through all the disc shows that the influence of the velocity gradient on the line formation is not very strong. The commonly used approximation, where the radiative transfer is solved through a static disc and the velocity is only included in the final flux integration, is sufficient. However, the validity of this approach must be checked for cases where discs are very cold, relatively transparent, and seen under very high inclinations.

This conclusion appears to be too general at this point because only three cases are investigated. We chose an SS Cyg model as an example of a typical CV disc. The quiescence phase is investigated since the disc is optically thinner than in the outburst. As an extreme case, a disc of an AM CVn system is chosen. The disc is colder here, the helium line is relatively narrow, and the inner edge of the disc is close to the WD, so that the maximal rotation velocity reaches $3360 \mathrm{~km} \mathrm{~s}^{-1}$ (SS Cyg $2700 \mathrm{~km} \mathrm{~s}^{-1}$ ). Nevertheless, the disc in this case is very geometrically thin. The angle between the midplane of the disc and its boundary $(\theta)$ is at most $1^{\circ}$. This geometry lowers the gradient of velocity along the line-of-sight. However, this effect is not very important. It is only six times lower for $i=80^{\circ}$ in comparison to the typical value $\theta \sim 5^{\circ}$. Considering very high rotation velocity, an AMCVn system remains a good subject for a test of the velocity shear effect.

Our calculations show another important result. The detailed radiative transfer with a precise inclusion of the velocity field must be solved for systems where we see only the disc rim. In this case, if the radiation transfer is solved through the static disc, only the value of velocity at the edge boundary (the lowest one) is included in the flux integration. To reflect all the velocity distribution, the velocity field must be taken into account in the radiative transfer. Even if the radiation from this direction is about one order of magnitude weaker than at the face-on view, it can be important for the study of self-shielding discs (e.g. some UX UMa or SW Sex stars). The velocity shear effect can also be important for eclipsing $\mathrm{CVs}$, since we see discs of these systems under high inclination angles. The technique presented here can therefore be very useful for studying these systems. To construct the disc map, it is necessary to know a limb-darkening law. Since we finally solve the radiative transfer in a $2 \mathrm{D}$ grid throughout the 
disc structure, the evaluation of this effect (Fig. 8, right panel) is more accurate than when only solving through the vertical structure of the disc.

Acknowledgements. We would like to thank the referee for the comments This research is partly financed by grants 228398 (the European Commission), 205/09/P476 (GA ČR), M100030973 (AV ČR), and LC06041 (MŠMT ČR) The Astronomical Institute Ondřejov is supported by project AV0Z10030501. V.S. acknowledges support by the Deutsche Forschungsgemeinschaft (DFG) through project C7 of SFB/Transregio 7 "Gravitational Wave Astronomy" and by Russian Basic Research Foundation (RFFI grant 09-02-97013-p-povolzh'e-a).

\section{References}

Adam, J. 1990, A\&A, 240, 541

Bath, G. T., Evans, W. D., Papaloizou, J., \& Pringle, J. E. 1974, MNRAS, 169, 447

Bitner, M. A., Robinson, E. L., \& Behr, B. B. 2007, ApJ, 662, 564

Cannon, C. J. 1970, ApJ, 161, 255

Hellier, C. 2001, Cataclysmic Variable Stars (Springer)

Horne, K., \& Marsh, T. R. 1986, MNRAS, 218, 761

Hubeny, I., \& Hubeny, V. 1997, ApJ, 484, 37

Hummel, W., Horne, K., Marsh, T., \& Wood, J. H. 1997, ASPC, 121, 498

Israel, G., \& Dall'Osso, S. 2009, ASSL, 359, 281

Knigge, Ch., Woods, J. A., \& Drew, J. E. 1995, MNRAS, 273, 225

Korčáková, D., \& Kubát, J. 2005, A\&A, 440, 715
Kromer, M., Nagel, T., \& Werner, K. 2007, A\&A, 475, 301

Kříž, S., \& Hubený, I. 1986, BAICz, 37, 129

Kunasz, P., \& Auer, L. H. 1988, JQSRT, 39, 67

Marsh, T. R. 1987, MNRAS, 228, 779

Mihalas, D., Kunasz, P. B., \& Hummer, D. G. 1976, ApJ, 206, 515

Morales-Rueda, L., \& Marsh, T. R. 2002, MNRAS, 332, 814

Nagel, T., Dreizler, S., Rauch, T., \& Werner, K. 2004, A\&A, 428, 109

Nagel, T., Rauch, T., \& Werner, K. 2009, A\&A, 499, 773

Nasser, M. R., Solheim, J. E., \& Semionoff, D. A. 2001, A\&A, 373, 222

Nelemans, G. 2005, ASPC, 330, 27

Papkalla, R. 1995, A\&A, 295, 551

Puebla, R. E., Diaz, M. P., \& Hubeny, I. 2007, AJ, 134, 1923

Rauch, T., \& Deetjen, J. L. 2003, ASPC, 288, 103

Rybicki, G. B., \& Hummer, D. G. 1983, ApJ, 274, 380

Schreiber, M. R., \& Lasota, J.-P. 2007, A\&A, 473, 897

Shakura, N. I., \& Sunyaev, R. A. 1973, A\&A, 24, 337

Smak, J. 1969, AcA, 19, 155

Smak, J. 1981, AcA, 31, 395

Sobolev, V. 1946, Dvizhushchiesia obolochki zvedz, Leningr. Gos. Univ., Leningrad

Schwarzenberg-Czerny, A., \& Rózyczka, M. 1977, AcA, 27, 429

Steffen, M. 1990, A\&A, 239, 443

Suleimanov, V. F. 1992, SvAL, 18, 104

Wade, R. A., \& Hubeny, I. 1998, ApJ, 509, 350

Warner, B. 1986, MNRAS, 222, 11

Warner, B. 1987, MNRAS, 227, 23

Werner, K., \& Husfeld, D. 1985, A\&A, 148, 417

Werner, K., Deetjen, J. L., Dreizler, S., et al. 2003, ASPC, 288, 31

Wood, M. A. 1995, Lecture Notes in Physics (Springer), 443, 41 